\title{
QoS Enabled Routing Protocol for CR MANET
}

\author{
Yan Sun, Chris Phillips \\ Queen Mary University of London \\ School of Electronic Engineering and Computer Science, \\ Mile End Road, London, UK \\ Yan.sun@qmul.ac.uk,Chris.phillips@qmul.ac.uk
}

\author{
Jingwen Bai, Liumeng Song, Rina $\mathrm{Wu}$, \\ Queen Mary University of London \\ School of Electronic Engineering and Computer Science, Mile \\ End Road, London, UK \\ jingwen.bai@qmul.ac.uk, liumeng.song@qmul.ac.uk, \\ ee08b361@qmul.ac.uk
}

\begin{abstract}
This paper proposes a cross layer routing protocol which operates with Cognitive Radio (CR) Mobile Ad Hoc Networks. In the near future, with the rapid development of hardware and chipset functionality, devices with CR capability will offer promising opportunities within future mobile networks. The new routing protocol exploits the transfer of CR performance data from the Physical/MAC layers up to Network layer as contributing factors within the route selection algorithms, which is the first time that available channel condition is considered within the Network layer. The performance of the proposed protocol is investigated via simulations and the results confirm its favorable operation within ad hoc network environments.
\end{abstract}

Index Terms - Cognitive Radio, MAC layer, Multi-channel, Internet of things, cross layer, routing

\section{INTRODUCTION}

Recent years, research into the Internet of Things (IoT) has developed significantly. No longer limited to the application concept phase, the IoT now encompasses access networks (such as sensor, RFID, and mobile devices), the backbone network structure (such as cloud computing, ubiquitous computing), as well as middleware techniques, embedded system control, IoT protocols, IoT signal processing, and IoT security and authentication. In all of these new domains the target is the same, which is to establish a healthy, robust and secure network for device-to-device communication. Meanwhile Cognitive Radio (CR) [1] has been identified as one of the promising techniques that is being adopted within wireless research to improve the utilization of scarce spectrum resources. Along with the fast evolution of hardware, it can be foreseen that in the near future, every device could be CR enabled. Nowadays, more and more IoT application scenarios are being studied, not only within restricted geographical areas, such as e-home or e-office, but also with regard to certain communication situations, such as vehicle-to-vehicle, emergency, disaster rescue, etc. Ad hoc networks, with their independence from pre-defined network infrastructure, are recognized as a popular approach for IoT. As long as the nodes in the ad hoc network are equipped with the self-organized conversation capability, a sufficient device-to-device communication network can be auto deployed anywhere on demand.

Considerable research work relating to $\mathrm{CR}$ networks has been carried out on the physical layer, such as in [2] [3] and the
MAC layer [4][5], CR performance is improving. However, another key issue in CR ad hoc networks regarding device-todevice communication, which cannot be avoided, is how to forward the data from the source to the destination efficiently. AODV (Ad hoc On_Demand Distance Vector) [6] and DSR (Dynamic Source Routing) [7] are regarded as the most widely deployed routing protocols in ad hoc networks. A few works have now started to consider new routing protocols for use in $\mathrm{CR}$ ad hoc networks. Work in [8][9] defined a SARP (Spectrum-Aware Routing Protocol) which assumes the mobile devices possess multi-RF interfaces. One geographically based routing protocol for $\mathrm{CR}$ ad hoc networks is proposed in [10]. Considering the device cost, more than one RF interface is not a cost effective choice and even with embedded GPS chipset, obtaining precise and up-to-date location information is still hard to achieve. Therefore in this paper, we propose a novel cross layer routing protocol in CR mobile Ad Hoc Networks (CR MANET) for IoT. With perfect knowledge of frequency usage data from the Physical layer, the Network layer works efficiently together with MAC layer to provide suitable path selection and multi-channel allocation for data forwarding and transmission. Furthermore, the cross layer design ensures that the routing protocol adapts well to the real-time radio environment throughout the whole transmission session.

The rest of the paper is organized as follows: Section II gives the overall cross layer design. How the CR is proposed to contribute during the route configuration phase is introduced in detail. Mobility handling in this new routing protocol is also addressed in this section. The implementation and simulation results are evaluated in Section III and conclusions are presented in Section IV.

\section{CROSS LAYER SYSTEM DESIGN}

\section{A. Cross layer protocol design}

In a conventional ad hoc network, a route finding phase will be invoked before a real data transmission starts. The intention of this phase is to establish the most suitable route between the source and destination according to the adopted routing protocol. By applying AODV and DSR, the shortest path can be discovered. If ABR (Associativity-Based Routing) [11] is used, the most stable path will have higher priority than the shortest path. In this paper, the proposed routing protocol takes suitable sub-channel information of each hop into account. 
With the sensing capability of each node, the condition of all the sub-channels between two neighbors will be fully captured and this information will be passed from the Physical layer to MAC and Network layers via the cross layer mechanisms in each node. In summary:

- Periodic sensing is carried out by the Physical layer scheduled by MAC layer in each node among the CRMANET. The sensing results are kept in the MAC layer and updated after each sensing cycle.

- Upon receiving a route establishment request from a source node, based on the latest sensing results, the MAC layer of each intermediate node will first sort all available channels into a prioritized list according to a QoS based algorithm then pass the prioritized channel list together with sensing results to the Network layer of the same node.

- Upon receiving the information from the MAC layer, the values of each entry in the neighbor table of each intermediate node are updated accordingly. Besides the entries introduced in AODV (such as destination, next hop, number of hops) and in ABR (such as number of stability ticks), the channel conditions (such as SNR, delay) will also be added into the neighbor table for route selection purposes.

- The intermediate node then attaches this additional information into the route request and broadcasts it to all its neighbors. It is rebroadcast until it reaches the destination.

- When the destination node receives a route establishment request message from its neighbors for all potential routes received within a specific period it invokes the route selection algorithm which considers the channel conditions, node stability and number of hops. The best route is selected by the destination and the route establishment response is sent back to the source node along the selected route.

- As sensing is a periodic activity at each node, the MAC layer of each node on the selected route will auto adapt the channel selection according to changes in the radio environment for data forwarding via the selected route from the source to the destination.

- If a link breaks during the conversation period, a local path recovery procedure is initiated similar to ABR [11]. If no alternative route is discovered locally, the source node will invoke a route request again in order to discover a new path.

Figure 1 illustrates the cross layer structure and the interaction between layers.

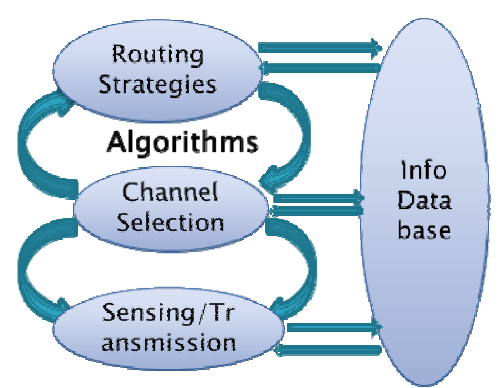

Figure 1 Cross Layer Routing Protocol Structure

\section{B. MAC layer Sub-channel Selection Schemes}

Let $n_{i}$ denote a single mobile node while $\mathrm{N}$ is the set of all nodes in the CRMANET, then $n_{i} \in N, \mathrm{i}=1,2, \cdots \mathrm{m}$.

Let $c_{j}$ be one sub-channel while $\mathrm{C}$ is the set for all subchannels belonging to one node, then $c_{j} \in C, \mathrm{j}=0,1, \cdots \mathrm{k}$

Let $S_{r}$ denote the one sensing result while $\mathrm{S}$ is the set of all sensing results, then $s_{r} \in S, \mathrm{r}=1,2, \cdots$, for instance, $s_{1}=$ receiving signal strength, $S_{2}=$ receiving noise strength, $S_{3}=$ delay, etc,

For all nodes, sub-channel 0 is set as the control channel and all other sub-channels are traffic channels.

The matrix of sensing results is shown as below:

$$
\left(\begin{array}{ccc}
n_{1 c_{0}}^{S} & \cdots & n_{m c_{0}}^{S} \\
\vdots & \ddots & \vdots \\
n_{1 c_{k}}^{S} & \cdots & n_{m c_{k}}^{S}
\end{array}\right)
$$

$n_{i c_{j}}^{S}$ represents all the sensing results of sub-channel $\mathrm{j}$ of node $n_{i}$

So the corresponding sensing results for one node are:

$$
\left\{n_{i c_{0}}^{S}, n_{i c_{1}}^{S}, n_{i c_{2}}^{S}, \cdots, n_{i c_{j}}^{S}, \cdots, n_{i c_{k}}^{S}\right\} \in n_{i C}^{S}
$$

As the sensing is periodically performed by the Physical layer of every node in the CRMANET, the MAC layer filters out available channels corresponding to every neighbor node and saves the results into the neighbor table in order of channel quality priority. The corresponding SNR and Delay values for each sub-channel pair are also calculated and saved in the neighbor tables. Let $S N R_{c_{j}}^{i k}$ denote the SNR of sub-channel $c_{j}$ between node $\mathrm{i}$ and node $\mathrm{k}$.

$$
S N R_{c_{j}}^{i k}(d B)=10.0 \times \log 10\left(\frac{P_{r c v d}^{k}}{N_{a c c u m}^{i k}+N_{b k g}^{i k}}\right)
$$


where $P_{r c v d}^{k}$ is the received power at node $\mathrm{k}, N_{\text {accum }}^{i k}$ is the accumulated noise and $N_{b k g}^{i k}$ is the background noise.

Delay $_{c_{i}}=$ transmission_delay $_{c_{i}}+$ propagation_delay $_{c_{i}}+\mathrm{buffer} \_$delay $_{c_{i}}$ while transmission_delay ${ }_{c_{j}}^{i k}=\frac{p k_{-} \text {size }}{t x_{\text {rate }}}$, where $p k_{-}$size is length of packet and tx_rate is transmission rate. and propagation_delay $y_{c_{j}}^{i k}=\frac{\text { prop_dis }}{\text { prop_velocity }_{\text {vel }}}$, w prop_velocity here prop_dis is propagation distance, and is velocity of light and buffer_delay $y_{c_{i}}$ is the time that a packet stays in corresponding queue buffer.

Let $S N R_{\text {th }}$ denote the threshold of SNR, let $C_{\text {avail }}$ denote the set of all available sub-channels, which can be used to send data packets, and let $W_{\mathrm{c}_{j}}$ denote the weighted value for computing sensing result of sub-channel $\mathrm{C}_{j}$.

$W_{\mathrm{c}_{j}}=\alpha \times S N R_{c_{j}}+\beta \times$ Delay $_{c_{j}}$, where $\alpha$ and $\beta$ are both weights and we have $\alpha+\beta=1$

Let $W_{t h}$ denote the threshold

$$
\text { If } W_{\mathrm{c}_{j}} \geq W_{t h}, c_{j} \in C_{\text {avail }} \text {. }
$$

All sub-channels in $C_{\text {avail }}$ are sorted in decreasing order of their weighted value $W_{\mathrm{c}_{j}}$. The sorting result can be taken as priority list, and is denoted by $C_{\text {sort }}$.

According to QoS scheduling, the MAC layer selects correspondence channels from top of priority list $C_{\text {sort }}$.

Right after each sensing, each node will update its "neighbor table" contents. Therefore the SNR and delay of each sub-channel will be recalculated, and ${ }^{W_{\mathrm{c}_{j}}}$ will be updated correspondingly. Therefore the priority list $C_{\text {sort }}$ will be automatically refreshed ready for the next packet transmission.

\section{Route selection scheme and algorithm}

The Route Request Broadcast Query (RRBQ) is generated by the source node $\mathrm{S}$. When it is propagated towards the destination, every node, other than the destination node, will add the following information to the message before rebroadcasting it:

- $\mathrm{n}_{\mathrm{ij}}(\mathrm{CHAN})$ denotes number of available sub-channels between node $n_{i}$ and node $n_{j}$

- $\mathrm{n}_{\mathrm{ij}}$ (TICKS) denotes the associativity ticks of each neighbor for this node.
The route selection algorithm is run at the destination. The destination node, instead of immediately responding to the first RRBQ message that arrives, it waits for a certain time for other possible RRBQ messages to reach it via different routes. The destination will then follow the two steps below to achieve the final selected route from source to destination:

Step One: The destination will generate three lists from one RRBQ message, as described below,

- IP list: $\left\{\mu_{\mathrm{n}_{\mathrm{i}}}, \mu_{\mathrm{n}_{\mathrm{i}}}, \ldots, \mu_{\mathrm{n}_{\mathrm{i}}}\right\}$, Where $\mu_{\mathrm{n}_{\mathrm{i}}}$ denotes the IP address of node $n_{i}$, $i \in \forall(1,2, \ldots m)$,

- Channel Availability list: $\left\{\mathrm{n}_{\mathrm{ij}}\right.$ (CHAN), $\left.\mathrm{n}_{\mathrm{ij}}(\mathrm{CHAN}) \ldots \mathrm{n}_{\mathrm{ij}}(\mathrm{CHAN})\right\}, \mathrm{i}, \mathrm{j} \in \forall(1,2, \ldots \mathrm{m})$

- Associativity Ticks list: $\left\{\mathrm{n}_{\mathrm{ij}}(\mathrm{TICKS}), \mathrm{n}_{\mathrm{ij}}(\mathrm{TICKS}) \ldots\right.$ $\left.\mathrm{n}_{\mathrm{ij}}(\mathrm{TICKS})\right\}, \mathrm{i}, \mathrm{j} \in \forall(1,2, \ldots \mathrm{m})$

Step Two: Finding the selected path as described below:

Let $\delta$ denote the total number of possible RRBQ messages from source node $\mathrm{S}$ to destination node $\mathrm{D}$,

Let $\partial$ denotes the $\partial$ th RRBQ message received by a destination in a given round of route discovery and $\varepsilon_{\partial}$ denotes number of hops for this route $(\partial=1,2, \ldots, \delta)$,

Let $\theta_{\partial}$ (CHAN) denote the mean value of the available channels per hop for each RRBQ, where

$$
\theta_{\partial}(\mathrm{CHAN})=\frac{\sum_{\varepsilon_{\partial}} \mathrm{n}_{\mathrm{ij}}(\mathrm{CHAN})}{\varepsilon_{\partial}}
$$

Let $\theta_{\partial}$ (TICKS) denote the mean value of the available channels per hop for each RRBQ, where

$$
\theta_{\partial}(\mathrm{TICKS})=\frac{\sum_{\varepsilon_{\partial}} \mathrm{n}_{\mathrm{ij}}(\mathrm{TICKS})}{\varepsilon_{\partial}}
$$

The selected route, denoted as Path $\rho$, should have the greatest mean number of available channels per hop, as shown below,

$$
\text { Path } \rho=\max \left\{\theta_{1}(\mathrm{CHAN}), \theta_{2}(\mathrm{CHAN}), \ldots, \theta_{\delta}(\mathrm{CHAN})\right\}
$$

But if $\theta_{\partial}(\mathrm{CHAN}), \partial=1,2, \ldots, \delta$ are all equal to each other, then the path with largest mean value of associativity ticks per hop ( i.e. the most stable route) should be the preferred choice, as shown below

$$
\text { Path } \rho=\max \left\{\theta_{1}(\mathrm{TICKS}), \theta_{2}(\mathrm{TICKS}), \ldots, \theta_{\delta}(\mathrm{TICKS})\right\}
$$

However, if $\theta_{\partial}$ (TICKS), $\partial=1,2, \ldots, \delta$ are all the same as well, the selected route should be the one with minimum hop count, as shown below,

$$
\text { Path } \rho=\min \left\{\varepsilon_{1}, \varepsilon_{2}, \ldots, \varepsilon_{\delta},\right\}
$$


As the route is selected by the destination D, a Route Reply (RR) message will be generated by D which contains the information below and will be unicasted back to $\mathrm{S}$.

IP list of the selected Path $\rho=\left\{\mu_{n_{i}}, \mu_{n_{i}}, \ldots, \mu_{n_{i}}\right\}$, Where $\mu_{n_{i}}$ denotes the IP address of node $n_{i}, i \in \forall(1,2, \ldots m)$,

Source IP address: $\mu_{\mathrm{S}}$

Destination IP address: $\mu_{\mathrm{D}}$

When an intermediate node receives the RR message, it updates its routing table making the route from source $\mu_{\mathrm{S}}$ to destination $\mu_{\mathrm{D}}$ valid and forwards the RR to the next upstream node. A valid route is established when the RR message reaches the source node $\mathrm{S}$.

\section{Cross-layer Routing Protocol Mobility Handling}

One of the features of mobile ad hoc networks is that nodes may exhibit random mobility and thus communication links are apt to be broken. In the $\mathrm{CR}$ enabled cross-layer routing protocol, a route reconstruction procedure is devised to cope with this. The principal is similar to ABR [11]

First of all, six definitions are introduced associated with the route reconstruction stage, as follows:

- BEACON_INTERVAL: indicates the frequency with which a node broadcasts a beacon signal.

- ALLOWED BEACON LOSS: the maximum number of continuously lost beacons before a link is considered broken.

- Recovery node: the originating node that sends out a Localized Query (LQ) to find an alternative partial route.

- LQ_TIMEOUT: the time that an LQ is regarded as alive in the network before it expires

- LQ_Retries: the maximum times LQ re-generation is permitted by one Recovery node.

- RN[DIR]: index DIR gives the direction of Route Notification (RN) message, RN[0] means downstream to destination, while $\mathrm{RN}[1]$ means upstream back to source

Secondly, the following three activities are defined for mobility handling:

- Connectivity Demonstration: an active path is used for data transmission, and the only way for all nodes along this path to demonstrate their activity and connectivity is periodic beaconing with their upstream and downstream nodes.

- Link Break Detection: if the ALLOWED_BEACON_LOSS is reached in one node from a specific neighbor, the link between these two nodes is treated as broken.

- Route Invalidation: Once the mobility-handling algorithm is triggered by link break detection, all paths impacted by the broken link are marked as invalid in the Route Notification (RN).
A moving Intermediate Node (IN) can cause frequent link breaks in MANET and to minimize the route discovery overhead, the broken path is better to be repaired locally. The procedure is as follows:

- The upstream originating node of the broken hop, (the first Recovery node), sends out a $\mathrm{RN}[0]$ to inform all the downstream nodes of the invalid path and a $\mathrm{RN}[1]$ to make SRC and all other upstream nodes buffer incoming packets temporarily.

- The first Recovery node broadcasts a Localized Query (LQ) to check whether the destination is within the connectivity range or not and at the same time instant, LQ_TIMEOUT starts counting down.

- If LQ_TIMEOUT is reached and no valid route is found, the same LQ will be sent out again until LQ_Retries is reached or a valid route to DEST is found.

- If LQ is sent out by the Recovery node and LQ_Retries reaches maximum times, but the destination is still unreachable then the recovery node will be traced back to the next upstream node.

- LQ is broadcast and forwarded to the DEST node. As with the BQ REPLY cycle, described in earlier work*, all intermediate nodes (INs) attach the additional information into LQ, including their IP address, subchannel availability and associativity ticks.

- DEST will wait for a certain period of time after it receives the first LQ to try to ensure that it receives all possible routes from Recovery node to DEST within the time slot. Then DEST will decide which path is the best one by the same route selection rules as the BQ_REPLY cycle in route discovery phase and send a REPLY packet back to the recovery node along the selected partial path.

- Upon the arrival of the REPLY within LQ_TIMEOUT by the recovery node, the broken route is reconstructed successfully by setting up a valid partial route. However, if REPLY does not come back within LQ_TIMEOUT and LQ_Retries is reached, the recovery node shall trace back to the next upstream node.

- When a break is detected an alternative route local recovery is initiated towards the SRC or the middle hop of the path depending on whether the break is upstream of the midpoint or not. If no alternative route can be found until the recovery node traces back to the middle hop of the path or the SRC, the SRC will be informed by $\mathrm{RN}$ to re-initiate route discovery.

All the above procedures are similar to those used in ABR [11] but the route selection algorithms are different as the CR sensing information is taken into consideration as described earlier. 


\section{SIMULATION AND EVALUATION}

\section{A. Simulation Topology and Scenario}

We adopt Opnet as the simulation platform. 802.11a is chosen as the wireless access technology for the Physical layer in the CRMANET supporting OFDM. The total bandwidth is $20 \mathrm{MHz}$ and there are 52 sub-channels with $312.5 \mathrm{kHz}$ per subchannel. In this simulation, we implement one sub-channel for control messages and four sub-channels for data transmission. Currently 802.11a does not support multiple channel scheduling in the MAC layer; so this functionality is added to the Opnet models. During the simulations, link breakages and local recoveries are introduced as well.

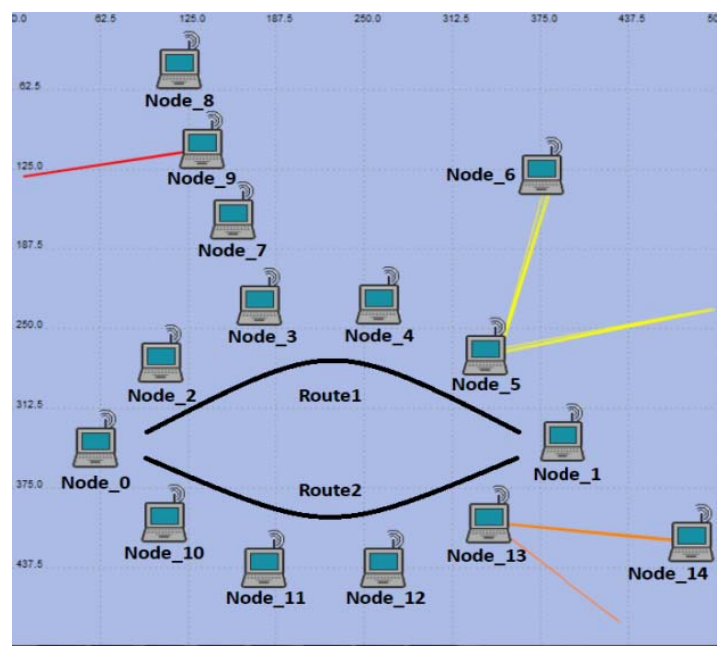

Figure 2 Topology with Node Movement

In this simulation scenario, there are two source-destination pairs: one is node_0 and node_1 and the other is node_7 and node_8 as shown in Figure 2. After every 5-minutes of transmission, node_5, node_9 and node_13 move out of connectivity range following the direction of the yellow, red and orange lines respectively with a speed of $20 \mathrm{~m} / \mathrm{s}$. At the same time, node_6 starts to move in towards node 5's previous location with the same speed. When node_6 arrives at the target position, it will stay there for 5 minutes. So does node_14 at node_13's previous location. Then node_6 and node_ 14 move back to their original location. At the same time, node_5 and node_13 start to move back and stay for another 5 minutes. The process repeats until the end of the simulation.

When node_9 reaches the location at the end of its orange trace, it will move back immediately. After it returns to its original location, which is between node_7 and node_8, it stays there for 5 minutes and then repeats the movement cycle.

We evaluate the proposed routing protocol by turning on/off the CR functions. It is easy to tell from the network topology shown in Figure 2 that from source node 0 to destination node_1, there are two options for the path selection, namely: Route1 via node $0-2-3-4-5-1$ and Route 2 via $0-10-11$ 12-13-1. The two scenarios we focus on are:
- CR Routing: As node_7 sends data to node_8 through node_9, and node_3 is within the transmission range of node 7 , then node 3 will experience interference from this node 7/8/9 transmission, reducing the number of available sub-channels. Therefore based on the periodic CR sensing results, Route2 has better available channel conditions compared to Route1 when the route selection algorithm is employed as described in Section II part D. Therefore Route2 is selected when $\mathrm{CR}$ is enabled for routing.

- No CR Routing, although all the nodes in Figure 2 act exactly the same as in the above CR Routing case, without $\mathrm{CR}$ sensing here, the affected sub-channel availability at node_3 will not be considered in the route selection algorithm at destination node_1. The adopted routing algorithm without $\mathrm{CR}$ sensing is the same as in ABR [11] which considers the stability and hop count for the best route selection. In the simulation, Route1 will be selected in this case.

\section{B. Simulation Parameter Settings}

TABLE I. TABLE 1 LinK BREAK SETtingS

\begin{tabular}{|c|c|}
\hline Items & Settings \\
\hline BEACON_INTERVAL & $1 \mathrm{~ms}$ \\
\hline ALLOWED_BEACON_LOSS & 5 \\
\hline LQ_TIMEOUT & $5 \mathrm{~ms}$ \\
\hline LQ Retries & 1 \\
\hline Node Movement Speed & $20 \mathrm{~m} / \mathrm{s}$ \\
\hline
\end{tabular}

Table 1 illustrates the parameter values for the link break functions in the simulation. There are two types of service simulated in this work: SM stands for Streaming service and BG stands for Background service. SM will demand 3 subchannels for each transmission while $B G$ will only need one sub-channel. The data generation ratio of SM and BG is 3:2. Each scenario runs for ten times with different random seeds. Each simulation lasts for 20 minutes. For clarity, only the mean values are shown in this section.

\section{Simulation Results}



Figure 3 Throughput at the Destination

Firstly the throughput at node_1 is monitored under the two routing schemes and the results are illustrated in Figure 3. The 
two services: $\mathrm{BG}$ and $\mathrm{BE}$ are both plotted under two different routing schemes. The results show that under CR Routing scheme, Route2 is chosen to serve more SM services which demands 3 sub-channels for each transmission. So priority of $\mathrm{SM}$ is guaranteed. However, the $\mathrm{BG}$ service has better throughput with Route1 under the No CR Routing scheme compared to Route 2 under the CR Routing scheme. The reason for this is that is without CR sensing assisting the route selection, Route1 cannot always guarantee three available subchannels for the SM service. So the BG service will have more sub-channels for data transmission while SM data is queuing in the buffer waiting for 3 sub-channels.

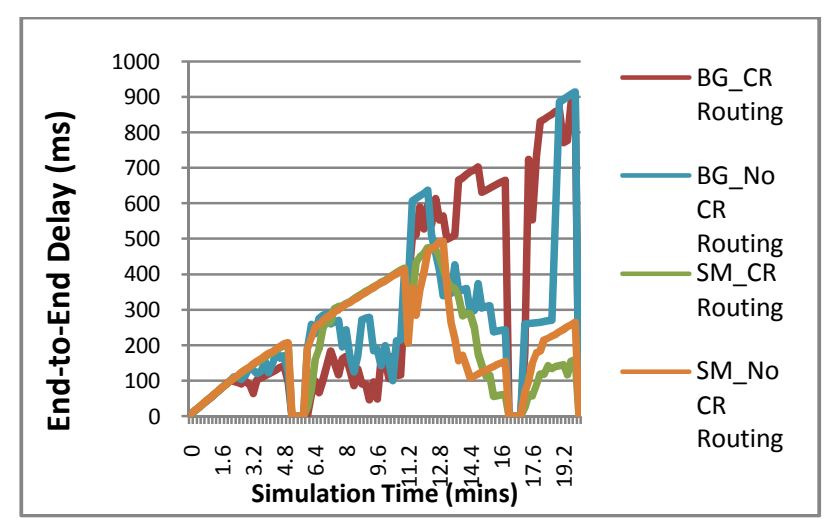

Figure 4 End-to-End Delay

The end-to-end delay is also investigated in this work. Again, for the SM service, CR Routing provides less delay compared to the No CR Routing case. Along with more BG data being transmitted in No CR Routing scheme, the delay is better for BG services when Routel is selected.

\section{CONCLUSION}

This paper proposes a cross layer routing protocol for CRMANET that allows the route selection algorithm to benefit from real time CR sensing data. Additionally the MAC layer is enhanced with a multi-channel selection capability exploiting information from radio sensing to adaptively compensate for the noisy environment. Simulations of two routing protocols, with/without CR sensing, are used to assess the performance of the proposed protocol. In general, the $\mathrm{CR}$ routing protocol can better understand the real time network situation therefore providing better network performance, especially for high priority services, such as SM. However, different QoS based sub-channel allocation schemes will affect the end users' experience which is now a topic for further investigation.

\section{REFERENCES}

[1] J. Mitola, III and G. Q. Maguire, "Cognitive radio: Making software radios more personal," IEEE Pers. Commun, vol. 6, pp. 13-18, 1999.

[2] Y . Zeng, Y.-C.Liang, "Covariance based signal detections for cognitive radio," IEEE DySPAN 2007, Dublin, Ireland, April 2007, pp202-207.

[3] A.Ghasemi, E.S.Sousa, "Optimization of spectrum sensing for opportunistic spectrum access in cognitive radio networks," IEEE Consumer Communications and Networking Conference (CCNC) 2007, Las Vegas, NV, USA, Jan 2007, pp 1022-1026

[4] H.Kim, K.G.Shin, "Efficient discovery of spectrum opportunities with MAC-layer sensing in cognitive radio networks," IEEE Transactions on Mobile Computing 7 (5) (2008) 533-545

[5] J.Jia, Q, Zhang, X.Shen, "HC-MAC: A hardware-constrained cognitive MAC for efficient spectrum management," IEEE Journal on Selected Areas in Communications 26 (1) (2008) 106-117

[6] C.E. Perkins and E.M. Royer. "Ad hoc On-Demand Distance Vector Routing." Proceedings of the 2nd IEEE Workshop on Mobile Computing Systems and Applications, New Orleans, LA, February 1999, pp. 90-100.

[7] D.B.Johnson, D.A.Maltz, J.Broch, "DSR: The Dynamic Source Routing Protocol for Multi-Hop Wireless Ad Hoc Networks," http://www.monarch.cs.cmu.edu

[8] S.Ju, J.B.Eveans, "Spectrum-Aware Routing Protocol for cognitive ad-hoc networks"in Proc. IEEE GLOBECOM 2009

[9] P.Kyasanur , N.H.Vaidya, "Routing in multi-channel multiinterface ad hoc wireless networks", Technical report, December 2004

[10] K..R.Chowdhury, M.D.Felice, "SEARCH: A routing protocol for mobile cognitive radio ad-hoc networks," December 2008, sumitted for publication

[11] C.K TOH, "Associativity-Based Routing for Ad-Hoc Mobile Networks", Wireless Personal Communications 4: 103-139, 1997. 